
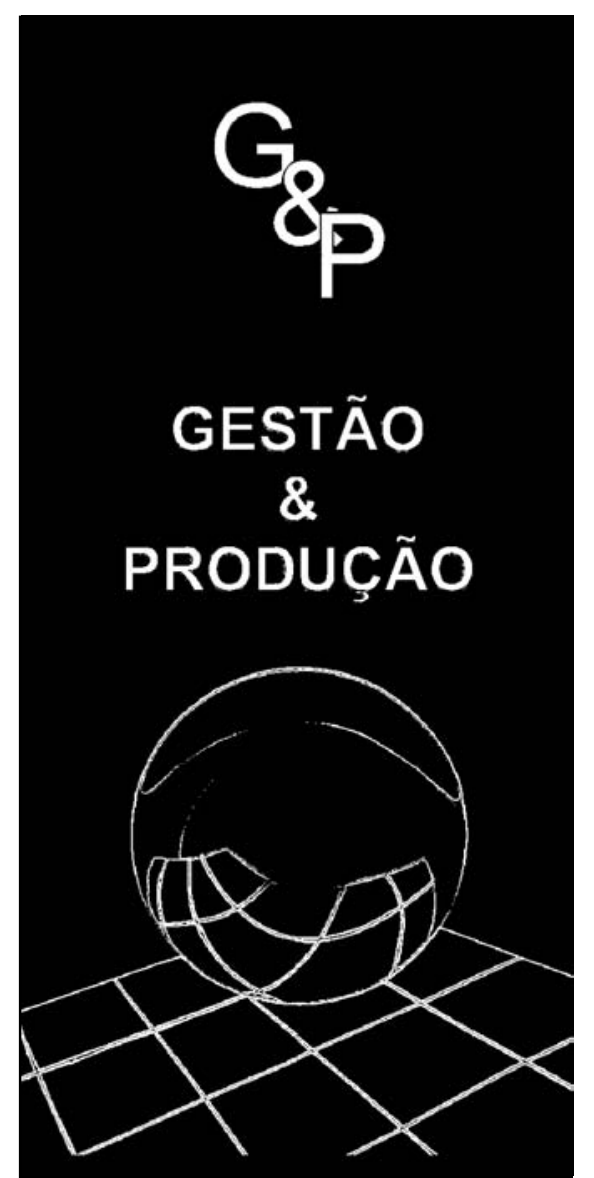

v.8, n.2, p.180-195, ago. 2001

\section{INTEGRAÇÃO DE MODELOS DE LOCALIZAÇÃO A SISTEMAS DE INFORMAÇÕES GEOGRÁFICAS}

\author{
Luiz Antonio Nogueira Lorena \\ Lab. Associado de Computação e Matemática Aplicada - LAC \\ Instituto Nacional de Pesquisas Espaciais - INPE \\ Edson Luiz França Senne \\ Departamento de Matemática \\ Faculdade de Engenharia de Guaratinguetá - FEG \\ Universidade Estadual Paulista - UNESP \\ João Argemiro de Carvalho Paiva \\ Divisão de Processamento de Imagens - DPI \\ Instituto Nacional de Pesquisas Espaciais - INPE \\ Marcos Antonio Pereira \\ Lab. Associado de Computação e Matemática Aplicada - LAC \\ Instituto Nacional de Pesquisas Espaciais - INPE
}

\title{
Resumo
}

O problema de p-medianas consiste em decidir onde localizar p centros em uma rede composta por vértices e arestas, de forma a minimizar a soma de todas as distâncias de cada vértice ao centro mais próximo. Em alguns casos, quando uma demanda estiver associada a cada vértice, pode haver restrições na capacidade de atendimento dos centros (problema de p-medianas com restrições de capacidade). Modelos de localização de facilidades têm sido propostos como ferramentas de auxílio à decisão, principalmente quando é possível usar Sistemas de Informações Geográficas (SIGs) na coleta e análise dos dados dos problemas. Apresentamos neste trabalho um relato da integração de modelos de p-medianas aos SIGs ArcView, da ESRI, e SPRING, um sistema desenvolvido no INPE. $O$ código que foi integrado a estes SIGs implementa uma abordagem recente da heurística Lagrangianal surrogate, onde a viabilização da solução dual é feita através de uma heurística de localizaçãoalocação alternada. $O$ trabalho apresenta alguns testes computacionais usando dados do município de São José dos Campos, com tamanhos variando até o máximo de 3280 vértices e 1141 centros, para o caso sem restrições de capacidade.

Palavras-chave: Problemas de Localização, Sistemas de Informações Geográficas, Heurísticas Lagrangianas. 


\section{Introdução}

$\mathrm{P}$ roblemas de localização tratam de decisões sobre onde instalar facilidades, considerando clientes que devem ser servidos de forma a otimizar algum critério (DREZNER, 1995; DASKIN, 1995). O termo "facilidades" é utilizado para designar fábricas, depósitos, escolas etc., enquanto "clientes" refere-se a depósitos, unidades de vendas, estudantes etc. Em geral, as facilidades podem ser selecionadas como novos centros a serem abertos ou escolhidas no conjunto de vértices existentes. Por isso, tais problemas também são conhecidos como problemas de localização-alocação, devido ao processo de alocação dos clientes aos centros abertos.

As aplicações de problemas de localização de facilidades ocorrem nos setores público e privado. No caso de setores públicos, procura-se maximizar a satisfação dos clientes em detrimento dos custos necessários para o alcance de tal objetivo. Localização de escolas, postos de saúde, corpo de bombeiros, ambulâncias, viaturas de polícia, pontos de ônibus, podem ser citados como exemplos de aplicações em setores públicos. No caso do setor privado, onde custos fixos estão envolvidos, as aplicações envolvem, em geral, fábricas, depósitos, torres de transmissão, lojas de franquias etc.

Em certos casos podem existir restrições sobre a capacidade de atendimento de tais centros. Neste tipo de problema, considera-se que cada cliente possui associada uma demanda a ser satisfeita pelo centro escolhido para atendêlo. A soma das demandas de todos os clientes atendidos por um centro não deve superar a capacidade de atendimento do mesmo. Quando esse tipo de condicionante estiver presente, dizemos tratar-se de um problema de localização com restrições de capacidade.

$\mathrm{O}$ problema de $p$-medianas é um problema clássico de localização de facilidades e consiste em localizar $p$ centros (medianas) em uma rede, de modo a minimizar a soma das distâncias de cada vértice ao centro mais próximo. As primeiras formulações deste problema foram apresentadas em HAKIMI $(1964,1965)$. O problema é bem conhecido como sendo NP-hard (GAREY \& JOHNSON, 1979). Vários métodos heurísticos e métodos que exploram busca em árvore têm sido desenvolvidos para o problema de $p$-medianas (TEITZ \& BART 1968; JARVINEN \& RAJALA, 1972; NEEBE, 1978; CHRISTOFIDES \& BEASLEY, 1982). Técnicas heurísticas de relaxação lagrangiana, combinadas a procedimentos de otimização por subgradientes, de um ponto de vista primal-dual, têm se mostrado eficientes na solução do problema (GALVÃO \& RAGGI, 1989; BEASLEY, 1993; LORENA \& SENNE, 1999).

Modelos de localização de facilidades têm sido propostos, há algum tempo, como ferramentas de auxílio à decisão, principalmente quando uma base de dados geograficamente referenciada estiver disponível. Nestes casos, os Sistemas de Informações Geográficas (SIGs) são muito importantes na coleta e análise desses dados (BURROUGH, 1986). Sistemas de Informações Geográficas (FISCHBECK, 1994) integram uma sofisticada interface gráfica a uma base de dados georreferenciados, constituindo-se em poderosas ferramentas de análise e planejamento espacial. Problemas complexos de localização de facilidades podem ser tratados com SIGs, considerando informações espaciais e socioeconômicas.

O uso combinado de SIGs e técnicas de Pesquisa Operacional para resolver problemas de localização ainda não está totalmente difundido na comunidade científica internacional. Mas, levando-se em conta sua capacidade de armazenar, exibir e manipular dados espacialmente distribuídos, a integração de algoritmos de localização aos SIGs foi iniciada há alguns anos.

Este trabalho relata a integração de modelos de localização de $p$-medianas, sem e com restrições de capacidade, aos SIGs ArcView da ESRI (Environmental Systems Research Institute, Inc.) e SPRING, um sistema desenvolvido pela Divisão de Processamento de Imagens (DPI) do INPE. O código integrado aos SIGs implementa uma abordagem recente da heurística lagrangiana/ surrogate, onde a viabilização da solução dual é 
feita através de uma heurística de localizaçãoalocação alternada. A integração ao ArcView foi feita através de scripts escritas em Аvenue e a integração ao SPRING foi realizada criando-se um método que atua na representação vetorial dos modelos de rede, temático e cadastral. $\mathrm{O}$ trabalho apresenta alguns testes computacionais usando dados do município de São José dos Campos, com tamanhos variando até o máximo de 3282 vértices e 1141 centros, para o caso sem restrições de capacidade.

\section{O Problema de $p$-Medianas}

$\mathrm{O}$ problemas de $p$-medianas considerados neste trabalho podem ser modelados como problemas de programação inteira 0-1. Sem perda de generalidade, vamos considerar que as medianas serão escolhidas do conjunto de vértices considerado no problema. Assim, para o caso sem restrições de capacidade, o modelo matemático será:

$$
\begin{gathered}
v(P)=\operatorname{Min} \sum_{i=1}^{n} \sum_{j=1}^{n} d_{i j} x_{i j} \\
P \text { sujeito a: } \sum_{j=1}^{n} x_{i j}=1, \quad \forall i \in N \\
\sum_{j=1}^{n} x_{j j}=p \\
x_{i j} \leq x_{j j}, \quad \forall i, j \in N, i \neq j \\
x_{i j} \in\{0,1\}, \quad \forall i, j \in N
\end{gathered}
$$

onde $N=\{1, \ldots, n\}$, sendo $n$ o número de vértices na rede; $p$ é o número de centros (medianas) a serem localizados; $\left[d_{i j}\right]_{n \times n}$ é uma matriz de custo (distância), com $d_{j j}=0, \forall j \in N$ e $\left[x_{i j}\right]_{n \times n}$ é a matriz de alocação, com:

$$
x_{i j}=\left\{\begin{array}{cc}
1, & \text { se o vértice } i \text { é atendido pelo } \\
0, & \text { centro } j, i \neq j ;
\end{array}\right.
$$

$$
x_{j j}= \begin{cases}1, & \text { se o vértice } j \text { é um centro; } \\ 0, & \text { caso contrário. }\end{cases}
$$

As restrições (2) e (4) garantem que cada vértice $i$ é alocado a somente um centro $j$, que deve ser uma mediana. A restrição (3) determina o número exato de medianas a serem localizadas $(p)$, e (5) corresponde às condições de integralidade. Para o caso com restrições de capacidade de atendimento $(P M C)$, as restrições (4) são substituídas por:

$$
\sum_{i=1}^{n} a_{i} x_{i j} \leq b_{j} x_{j j}, \quad \forall j \in N
$$

sendo $a_{i}$ a demanda do vértice $i$ e $b_{j}$ a capacidade de atendimento do centro $j$, se este for escolhido como mediana.

A técnica heurística usada para resolver $P$ de forma aproximada é conhecida como relaxação lagrangiana/surrogate e já foi aplicada com sucesso a outros problemas de otimização combinatória (LORENA \& LOPES, 1994; LORENA \& NARCISO, 1996; LORENA \& SENNE, 1996, 1999; SENNE \& LORENA, 1997; NARCISO \& LORENA, 1999). Uma discussão sobre as relaxações lagrangiana e surrogate pode ser encontrada em PARKER \& RARDIN (1988). A relaxação lagrangiana/ surrogate utilizada neste trabalho para $\mathrm{o}$ problema de $p$-medianas sem restrições de capacidade está descrita em SENNE \& LORENA (2000).

\section{A Heurística Lagrangiana/Surrogate}

A relaxação lagrangiana/surrogate desenvolvida para resolver de forma aproximada o problema de $p$-medianas com restrições de capacidade $P M C$ apresenta melhores resultados que a relaxação lagrangiana usual, obtendo limitantes de igual qualidade com menor esforço computacional (NARCISO \& LORENA, 1999; LORENA \& SENNE, 1999).

Considerando $\lambda \in \Re_{+}^{n}$, a restrição surrogate relativa às restrições (2) será: 


$$
\sum_{i=1}^{n} \sum_{j=1}^{n} \lambda_{i} x_{i j}=\sum_{i=1}^{n} \lambda_{i}
$$

e a relaxação lagrangiana/surrogate de $P M C$, considerando a variável dual $t \geq 0$, será:

$$
\begin{gathered}
v\left(L_{t} P M C^{\lambda}\right)=\operatorname{Min} \sum_{i=1}^{n} \sum_{j=1}^{n}\left(d_{i j}-t \lambda_{i}\right) x_{i j}+t \sum_{i=1}^{n} \lambda_{i} \\
L_{t} P M C^{\lambda} \quad \text { sujeito a: (3), (4') e (5). }
\end{gathered}
$$

Para $\quad t \geq 0$ e $\quad \lambda \in \Re_{+}^{n}$ dados, tem-se $v\left(L_{t} P M C^{\lambda}\right) \leq v\left(P M C^{\lambda}\right) \leq v(P M C)$, onde $P M C^{\lambda}$ representa a relaxação surrogate de $P M C$ (LORENA \& NARCISO, 1996). O problema $L_{t} P M C^{\lambda}$ pode ser resolvido considerando-se a restrição (3) implicitamente e decompondo-se o problema para o índice $j$, obtendo-se os seguintes $n$ subproblemas da mochila:

$$
\operatorname{Min} \sum_{i=1}^{n}\left(d_{i j}-t \lambda_{i}\right) x_{i j}
$$

sujeito às restrições (4') e (5). Cada subproblema é facilmente resolvido calculando-se:

$$
\beta_{j}=\sum_{i=1}^{n}\left[\min \left\{0, d_{i j}-t \lambda_{i}\right\}\right]
$$

e definindo-se $C$ como o conjunto de índices dos $p$ menores $\beta_{j}$. Assim, uma solução $x_{i j}^{\lambda}$ para o problema $L_{t} P M C^{\lambda}$ é dada por:

$$
x_{j j}^{\lambda}= \begin{cases}1, & \text { se } j \in C \\ 0, & \text { caso contrário }\end{cases}
$$

e, para todo $i \neq j$ :

$$
x_{i j}^{\lambda}= \begin{cases}1, & \text { se } j \in C \text { e } d_{i j}-t \lambda_{i}<0 \\ 0, & \text { caso contrário }\end{cases}
$$

Neste caso, o valor da solução lagrangiana/ surrogate é dado por:

$$
v\left(L_{t} P M C^{\lambda}\right)=\sum_{j=1}^{n} \beta_{j} x_{j j}+t \sum_{i=1}^{n} \lambda_{i}
$$

A solução $x^{\lambda}$ não é necessariamente viável, mas o conjunto $C$ identifica os vértices escolhidos como centros que podem ser usados para produzir soluções viáveis para ambos os problemas. Para o caso sem restrições de capacidade, os vértices são alocados aos centros mais próximos, produzindo uma solução viável $x^{f}$ para $P$ dada por:

$$
\left(x_{j j}^{\lambda}\right)^{f}= \begin{cases}1, & \text { se } j \in C \\ 0, & \text { caso contrário }\end{cases}
$$

e para todo $i \neq j$ :

$$
\left(x_{i k}^{\lambda}\right)^{f}= \begin{cases}1, & \text { se } k \text { tal que } d_{i k}=\min _{j \in C}\left\{d_{i j}\right\} \\ 0, & \text { caso contrário }\end{cases}
$$

$\operatorname{com} v^{f}=\sum_{i=1}^{n}\left[\min _{j \in C}\left\{d_{i j}\right\}\right]$.

Fazendo-se $t=1$ na relaxação $L_{t} P M C^{\lambda}$ tem-se a relaxação lagrangiana usual, considerando o multiplicador $\lambda$. Para um multiplicador $\lambda$ fixo, o melhor valor para $t$ pode ser encontrado resolvendo-se o problema dual lagrangiano: $v\left(D_{t}^{\lambda}\right)=\max _{t \geq 0} v\left(L_{t} P M C^{\lambda}\right)$.

O melhor valor da relaxação lagrangiana/ surrogate fornece um limitante melhor do que o obtido pela relaxação lagrangiana usual. Uma solução exata para $D_{t}^{\lambda}$ pode ser obtida por uma busca sobre diferentes valores de $t$ (MINOUX, 1975; HANDLER \& ZANG, 1980). Entretanto, em geral, existe um intervalo $t_{0} \leq t \leq t_{1}$ (com $t_{0}=1$ ou $\left.t_{1}=1\right)$ que também produz limitantes melhores, como pode-se ver pela Figura 1.

Assim, para obter um bom limitante, não é necessário encontrar o melhor valor de $t\left(t^{*}\right)$, sendo suficiente encontrar um valor $T$ tal que $t_{0} \leq T \leq t_{1}$, através de um procedimento de busca heurística (SENNE \& LORENA, 2000). Entretanto, se o valor de $T$ se mantiver constante por um número de iterações fixado a priori, então esse valor será mantido para todas as relaxações seguintes e o procedimento de busca não será mais executado.

O seguinte algoritmo de subgradientes é utilizado para resolver o problema de $p$-medianas: 


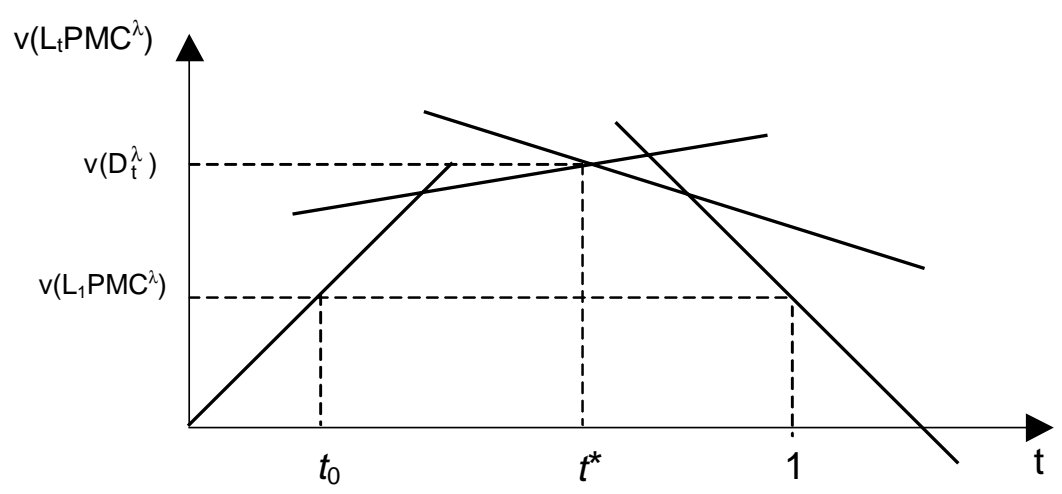

Figura 1 - Limitantes lagrangiano/surrogate.

\section{Algoritmo de Subgradientes:}

$\operatorname{Dados} \lambda \geq 0, \lambda \neq 0$;

Fazer $l b=-\infty, u b=+\infty, C=\varnothing$;

Repetir

Resolver a relaxação $L_{t} P M C^{\lambda}$ obtendo $x^{\lambda} \mathrm{e}$ $v\left(L_{t} P M C^{\lambda}\right)$;

Obter uma solução viável $x^{f}$ e seu valor $v^{f}$;

Atualizar $\quad l b=\max \left\{l b, v\left(L_{t} P M C^{\lambda}\right)\right\}$

$u b=\min \left\{u b, v^{f}\right\}$;

Fixar $x_{j j}=1$ se $v\left(\left.L_{t} P M C^{\lambda}\right|_{x j j=0}\right) \geq u b, j \in N-C$;

Atualizar o conjunto $C$;

Fazer $g_{i}^{\lambda}=1-\sum_{j=1}^{n} x_{i j}^{\lambda}, i \in N$

Atualizar o tamanho do passo $\theta$;

Fazer $\lambda_{i}=\max \left\{0, \lambda_{i}+\theta g_{i}^{\lambda}\right\}, i \in N$;

Até que (algum teste de parada seja satisfeito).

O valor inicial de $\lambda$ usado é dado por $\lambda_{i}=\min _{j \in N}\left\{d_{i j}\right\}, i \in N$. O tamanho do passo é calculado como:

$$
\theta=\frac{\pi(u b-l b)}{\left\|g^{\lambda}\right\|^{2}} .
$$

O controle do parâmetro $\pi$ é o proposto por HELD \& KARP (1971). Inicialmente seu valor é fixado em 2, sendo reduzido à metade em cada iteração sempre que $l b$ mantiver seu valor constante por 30 iterações sucessivas. Foram utilizados os seguintes testes de parada:

a) $\pi \leq 0.005$;

b) $u b-l b<1$;

c) $\left\|g^{\lambda}\right\|^{2}=0$;

d) todas as medianas foram fixadas.

\subsection{Algoritmo de Busca Local}

No caso com restrições de capacidade, o processo de obtenção de uma solução factível $x^{f}$ para o PMC utiliza o algoritmo $M T G H$ de MARTELLO \& TOTH (1990), para resolver de forma aproximada o seguinte problema generalizado de alocação $(P G A)$ :

$$
\operatorname{Max} z=\sum_{i \in N-C} \sum_{j \in C} p_{i j} x_{i j}^{f}
$$

$P G A$ sujeito a:

$$
\begin{array}{ll}
\sum_{i \in N-C} a_{i} x_{i j}^{f} \leq b_{j}, & \forall j \in C \\
\sum_{j \in C} x_{i j}^{f}=1, \quad & \forall i \in N-C \\
x_{i j}^{f} \in\{0,1\}, \quad \forall i \in N-C, \forall j \in C
\end{array}
$$

sendo $p_{i j}=-d_{i j}$ o ganho se o vértice $i$ for atribuído ao centro $j, i \in N-C$ e $j \in C ; a_{i}$ a demanda associada ao vértice $i, i \in N-C ; b_{j}$ a capacidade 
do centro $j, j \in C$ e $x_{i j}^{f}=1$, se o vértice $i$ é alocado ao centro $j \in C$ e $x_{i j}^{f}=0$, caso contrário.

A heurística de localização-alocação, inspirada nos trabalhos de COOPER (1963) e TAILLARD (1996), baseia-se na observação que, após a definição de $x^{f}$, obtém-se exatamente $p$ aglomerados (clusters) $C_{m}=\left\{j_{m}, I_{m}\right\}$, onde $j_{m} \in J$ é o índice do centro relativo ao cluster $C_{m}$, $m \in\{1,2, \ldots, p\}$, e $I_{m}=\left\{i \in N-C \mid x_{i j_{m}}^{f}=1\right\}$ é o conjunto dos vértices alocados ao centro $j_{m}$. A solução $x^{f}$ pode ser melhorada procurando-se por um novo centro dentro de cada cluster, trocandose a mediana atual por outro vértice e recalculando-se as alocações. Este processo se repete até que não seja mais possível obter melhorias no custo total da alocação. $O$ algoritmo de localização-alocação utilizado neste trabalho, para o caso capacitado, está dado a seguir.

\section{Heurística de Localização-Alocação (HLA)}

\section{Sejam:}

- $I_{m}$ o conjunto de índices dos pontos de demanda alocados ao centro $m$;

- $Z_{m}=\sum_{k \in I_{m}} d_{k j_{m}}$ o custo do cluster $C_{m}=\left\{j_{m}, I_{m}\right\}$;

- $D_{m}=\sum_{k \in I_{m} \cup\left\{j_{m}\right\}} a_{k}$ a demanda total do cluster $C_{m}$;

Repetir

(A)

Para cada cluster $C_{m}, m=1, \ldots, p$, fazer:

ntrocas $=0$;

Se (ntrocas < MAX_TROCAS) então:

Se existe um vértice $i \in I_{m}$ tal que:

$$
\begin{aligned}
& b_{i} \geq D_{m} \mathrm{e} \\
& Z_{m(i)}<Z_{m}, \text { onde } I_{m(i)}=I_{m} \cup\left\{j_{m}\right\}-\{i\} \\
& \text { Então }
\end{aligned}
$$

Intercambiar $i$ com $j_{m}$, atualizando o cluster $C_{m}$;

ntrocas $=$ ntrocas +1 ;
(B)

Seja $J=\left\{j_{1}, \ldots, j_{p}\right\}$ o conjunto atual de índices dos centros.

Resolver o $P G A$ considerando o conjunto $J$, obtendo um novo conjunto de clusters $C_{1}, \ldots, C_{p}$.

Enquanto (for possível melhorar a solução viável).

O algoritmo de localização-alocação acima considera ainda, nos pontos assinalados por (A) e (B), a possibilidade de alterações nas alocações dos vértices aos centros de cada cluster, verificando a possibilidade de um vértice $n_{r}$ pertencente a um cluster $C_{r}$ ser removido deste cluster e alocado a outro cluster $C_{s}(r \neq s)$, ou então verificando a possibilidade de dois vértices $n_{u}$ e $n_{v}$ (pertencentes aos clusters $C_{u}$ e $C_{v}$, respectivamente) serem intercambiados, levando-se em conta as demandas dos vértices, as capacidades dos respectivos centros e o custo da solução resultante. Nos testes computacionais foi utilizado MAX_TROCAS $=3$.

\section{Integração a Sistemas de Informações Geográficas}

algoritmo de p-medianas descrito na seção anterior foi integrado a dois Sistemas de Informações Geográficas: ArcView (ESRI, 1996) e SPRING (SPRING, 1998). Os estudos simularam a instalação de facilidades considerando uma base de dados reais de algumas regiões da cidade de São José dos Campos, SP.

As subseções a seguir contêm alguns detalhes das etapas das duas integrações desenvolvidas neste trabalho.

\subsection{Integração ao Arc View}

Os algoritmos para a solução dos problemas de $p$-medianas com e sem restrições de capacidade foram implementados utilizando a linguagem $\mathrm{C}$ e compilados com MS Visual C++. Os dados necessários aos programas foram 
obtidos a partir da base de dados dos temas (representação gráfica de um objeto geográfico, p. ex., ruas, quadras, imóveis etc.) disponíveis. Através de scripts desenvolvidas em Avenue, disponível no ArcView, esses dados foram organizados em arquivos texto para serem passados como entrada aos respectivos programas.

Em ambos os casos, a distância entre os vértices foi calculada a partir da escala do mapa no qual estão inseridos. Os valores resultantes representam a distância direta linear entre os vértices ou a distância sobre os arcos (ruas e avenidas) que compõem o mapa. Neste modelo de solução do problema de $p$-medianas, a distância entre os vértices foi o único parâmetro de custo considerado.

Para a visualização da solução, utilizou-se a função Spider, disponível no ArcView, que foi modificada para se adequar às necessidades da integração. Esta função verifica as distâncias entre os vértices de demanda, contidos em um tema, e os vértices relativos aos centros ofertantes, contidos em outro tema, e representa a alocação dos vértices aos centros selecionados para atendimento.

\subsubsection{Caso 1: $p$-medianas sem restrições de capacidade}

Foram desenvolvidos dois módulos para resolver o problema de $p$-medianas sem restrições de capacidade. $\mathrm{O}$ primeiro módulo utiliza como entrada os dados um arquivo texto gerado por uma script. Este arquivo contém no seu primeiro registro o número de vértices e o número de medianas a serem considerados. Os registros seguintes formam uma lista de coordenadas X-Y de todos os vértices do tema de pontos escolhido para estudo. Como resultado, o programa gera um arquivo tipo texto contendo a matriz simétrica de distâncias entre cada par de vértices.

O segundo módulo contém a implementação da heurística lagrangiana/surrogate. $\mathrm{O}$ arquivo de distâncias gerado pelo módulo anterior contém os dados necessários ao programa que, após o processamento, fornece a solução para o problema no formato de um arquivo tipo texto, contendo uma lista tripla formada por: vértice de demanda, seu centro correspondente e a distância entre eles. O último registro deste arquivo indica o status da solução encontrada: "Ótima" (gap fechado por limites) ou "NãoÓtima".

As Figuras 2 e 3 mostram alguns resultados obtidos utilizando dados do centro da cidade de São José dos Campos. Os polígonos de fundo correspondem ao tema escolhido para representar as quadras do centro da cidade. Os pontos sobrepostos são os vértices de demanda considerados.

A script desenvolvida para esta integração utiliza a informação do arquivo contendo a solução do problema e cria dois novos temas. Um tema de pontos representa as medianas encontradas pelo algoritmo, e um tema de linhas representa a alocação centro-vértice.

\subsubsection{Caso 2: p-medianas com restrições de capacidade}

Para resolução do problema de $p$-medianas com restrições de capacidade, apenas um módulo foi desenvolvido, contendo todas as rotinas do procedimento heurístico. Parâmetros específicos na linha de comando permitem selecionar entre distâncias lineares ou calculadas sobre o tema de rede.

Os valores de demanda considerados neste trabalho foram extraídos dos temas disponíveis, baseados no número de imóveis existentes em cada quadra, onde quadras com número nulo de imóveis recebiam um valor de demanda igual a 1 . A partir dessa informação, a script calcula a demanda total como sendo a soma da demanda de todos os vértices do tema de pontos selecionados. Este valor é então dividido pelo número de medianas a serem localizadas, definindo assim a capacidade de cada centro de atendimento. Para efeitos de estudo, este valor foi multiplicado por um fator $t>1$, permitindo modelar cenários com escassez ou excesso na capacidade de atendimento dos centros. Por questões de viabilidade, a 


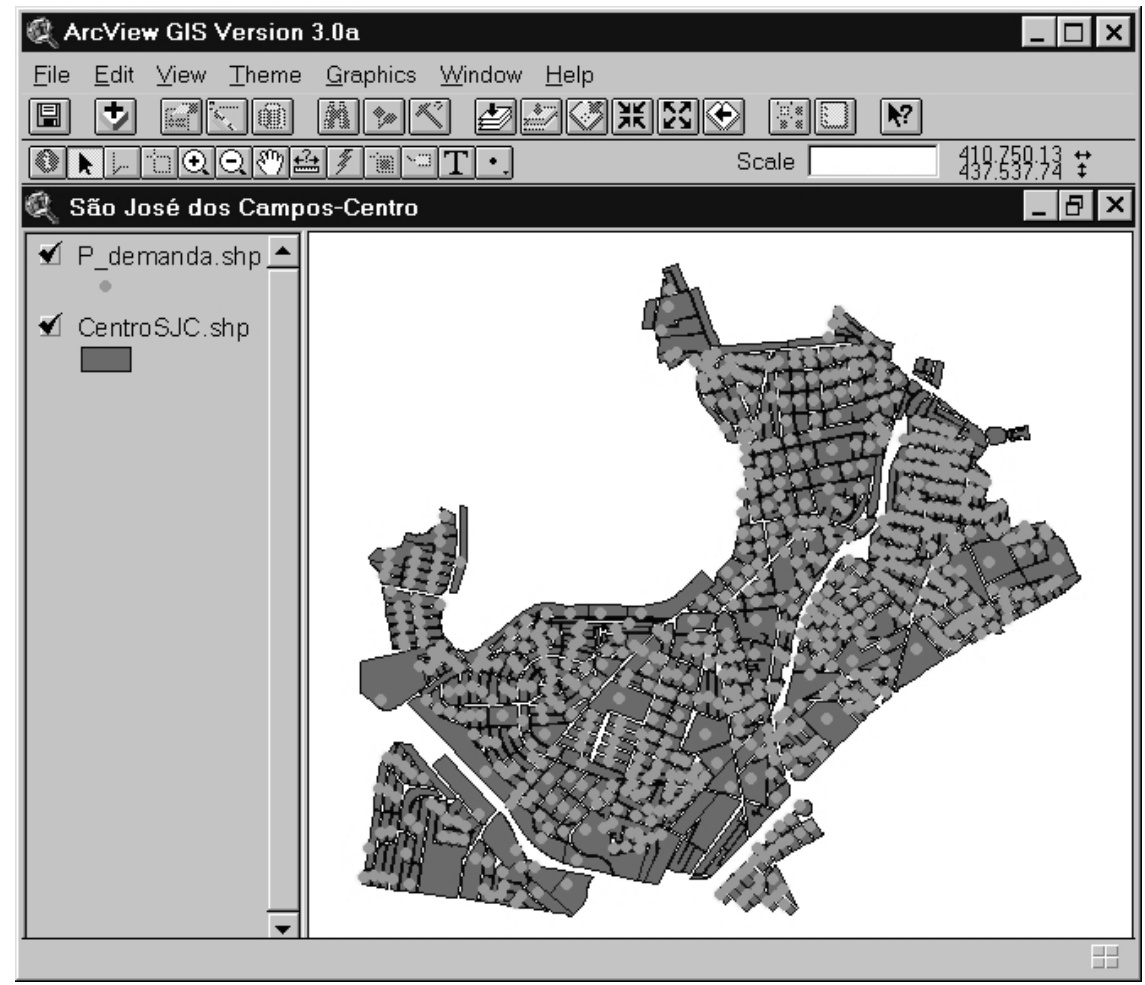

Figura 2 - Pontos de demanda no ArcView.

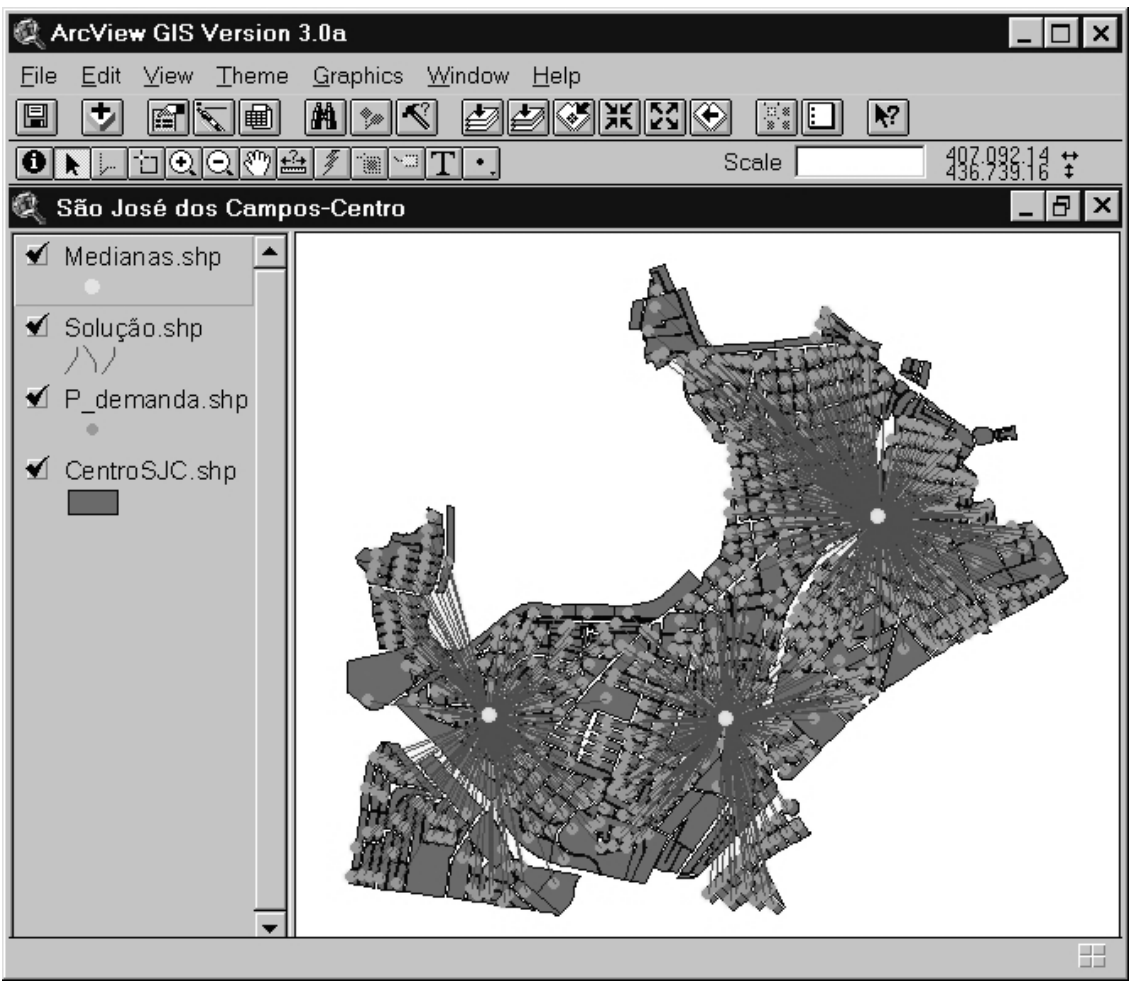

Figura 3 - Visualização da solução de problema de $p$-medianas. 
capacidade individual de atendimento de cada centro (considerada idêntica neste estudo) não deve ser inferior ao maior valor de demanda individual observado no tema escolhido.

O programa desenvolvido para resolver o problema de $p$-medianas capacitado utiliza como entrada de dados um arquivo tipo texto gerado pela script, que contém no seu primeiro registro o número de vértices e o número de centros a serem considerados. Dependendo do tipo de distância considerada (linear ou na rede), os registros seguintes formam uma lista ordenada de informações que possibilitam ao programa encontrar a solução, apresentada como um arquivo texto, contendo a alocação centro-vértice e a respectiva distância. Um número ao final do arquivo indica o tipo da solução obtida: "Ótima" ou "Não-ótima".

Nas Figuras 4 e 5 tem-se a visualização da solução de um problema contendo 31 vértices, dos quais foram selecionados 3 para a instalação de facilidades. No primeiro estudo foram consideradas distâncias lineares e no segundo foram utilizadas distâncias calculadas sobre a rede que representa um subconjunto das ruas que compõem o centro da cidade de São José dos Campos. Como se pode observar, existem diferenças entre as soluções dos dois estudos.

Observa-se que a formação de agrupamentos permite considerar a possibilidade de roteamento dos vértices associados a cada centro. Neste exemplo, considerou-se que o centro (em destaque) seria o ponto de origem e de destino da rota. Para o cálculo das rotas foi utilizado o módulo Network Analyst, que integra o pacote de módulos opcionais do ArcView.

\subsection{Integração ao SPRING}

O Sistema de Processamento de Informações Georreferenciadas (SPRING, 1998) é um sistema computacional desenvolvido pela equipe da Divisão de Processamento de Imagens (DPI) do Instituto Nacional de Pesquisas Espaciais. Este sistema tem por objetivo a integração e análise de diferentes tipos de dados espaciais.
O modelo de dados do SPRING está baseado no paradigma de orientação a objetos (CÂMARA, 1995). Um banco de dados geográfico é composto de planos de informação, de objetos geográficos, e de informações não espaciais. Os planos de informação podem representar informações contínuas no espaço (campos), ou os objetos geográficos individuais. Cada plano de informação pode conter representações espaciais do tipo vetorial ou varredura. A representação vetorial corresponde a linhas, pontos, e polígonos que definem as formas de representação dos objetos espaciais, enquanto a representação de varredura corresponde a uma matriz de pontos com valores em cada célula.

Os tipos de dados tratados no SPRING são:

- Mapas temáticos: cada informação representa um tema ou classe de informação. Por exemplo, as classes de uso do solo de uma região.

- Mapas cadastrais ou mapa de objetos: ao contrário de um mapa temático, cada elemento é um objeto geográfico que possui atributos e pode estar associado a várias representações gráficas. Por exemplo, os lotes de uma cidade são elementos do espaço geográfico que possuem atributos (dono, localização, valor venal, IPTU devido, etc.) e que podem ter representações gráficas diferentes (poligonais, lineares ou pontuais) em mapas de escalas distintas.

- Mapas de redes: correspondem a mapas cadastrais, com a diferença de que geralmente os objetos são representados por elementos lineares ou pontuais. As representações pontuais devem estar localizadas em pontos de intersecção de linhas na rede.

- Modelo numérico de terreno: denota a representação de uma grandeza que varia continuamente no espaço. Comumente associados à altimetria, podem ser utilizados para modelar outros fenômenos de variação contínua (como variáveis geofísicas, geoquímicas e batimetria).

- Imagens: representam dados de sensoriamento remoto ou fotografias aéreas. 


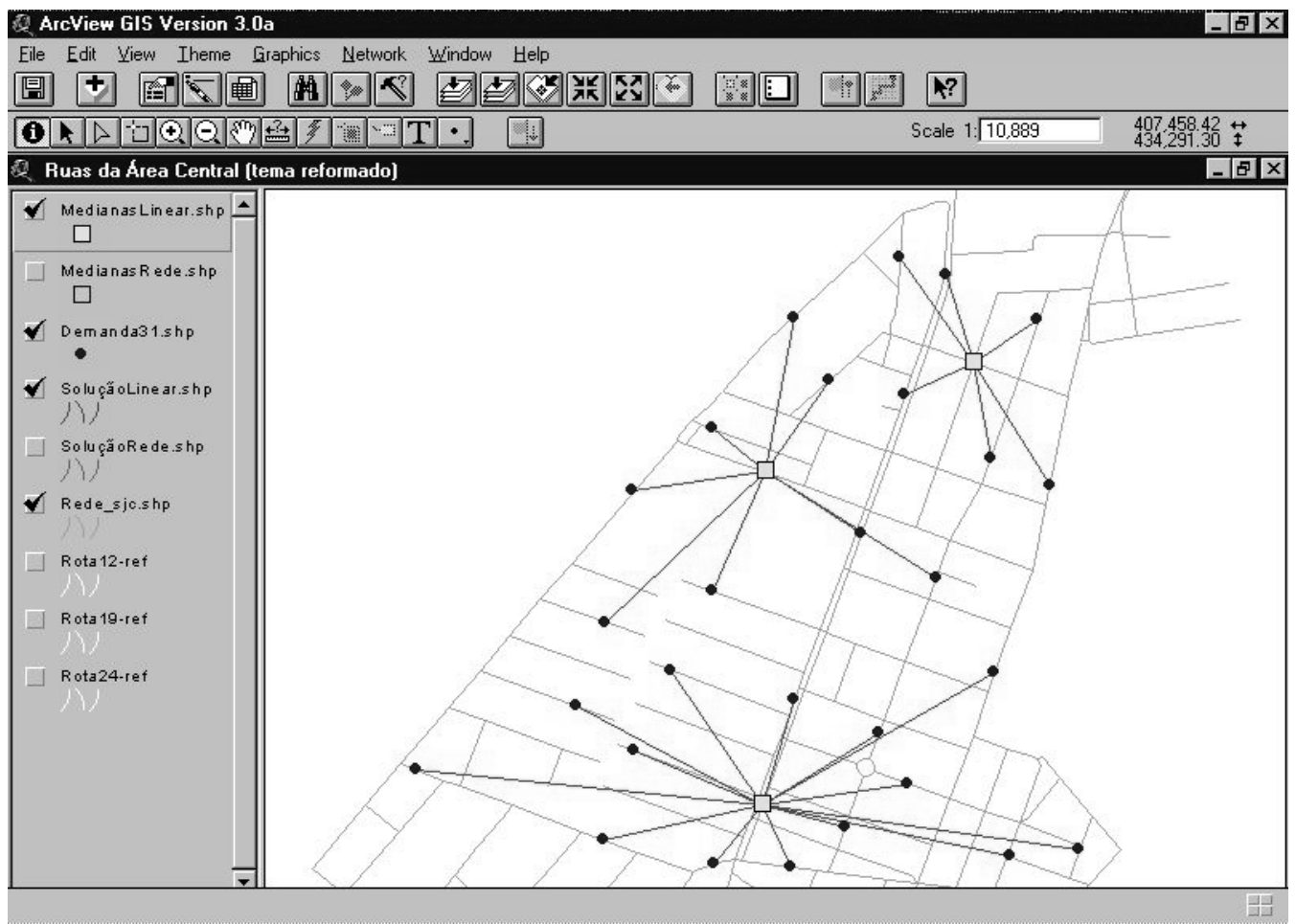

Figura 4 - Solução do problema de p-medianas com restrições de capacidade, distâncias lineares.

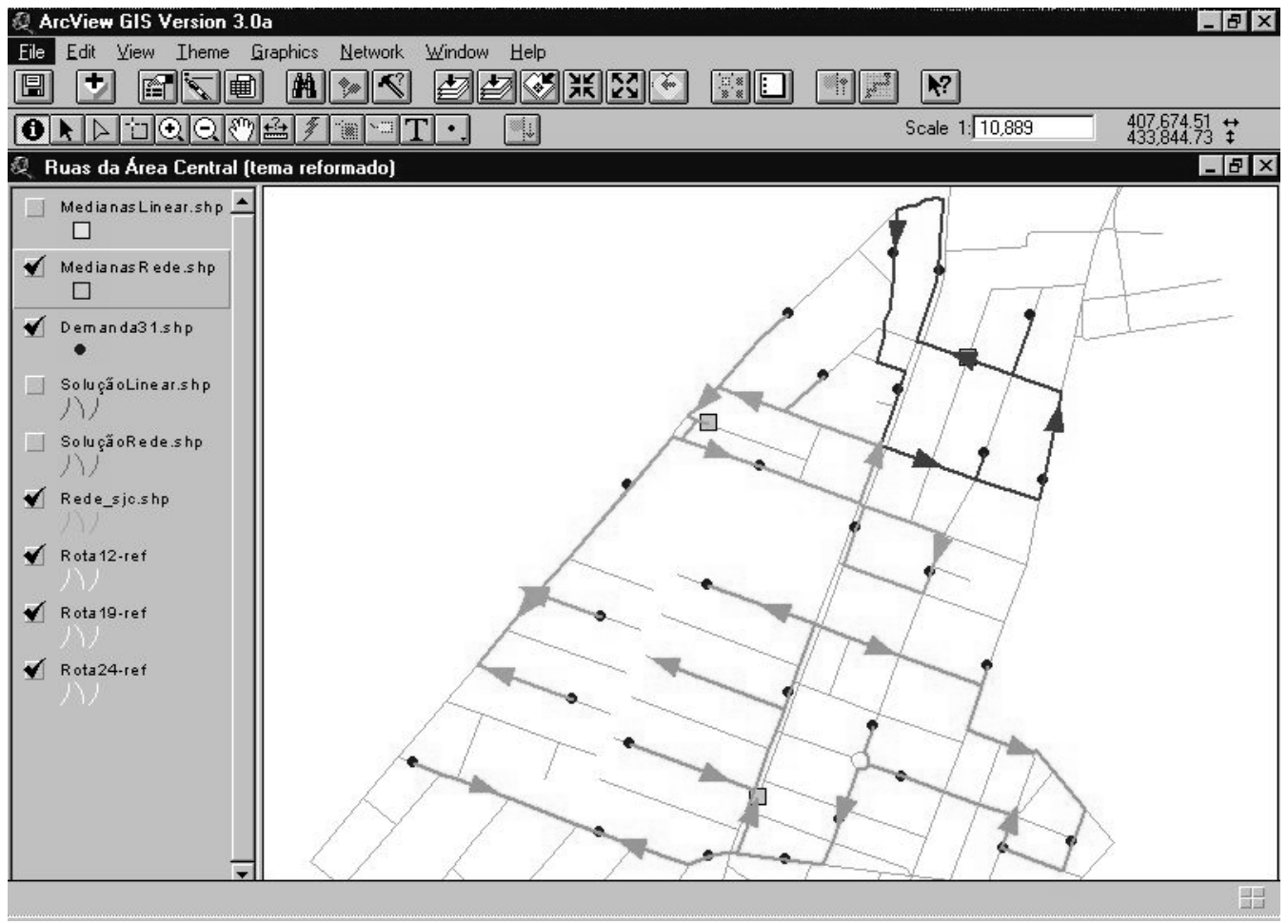

Figura 5 - Solução do problema de $p$-medianas com restrições de capacidade, com roteamento, considerando distâncias na rede. 


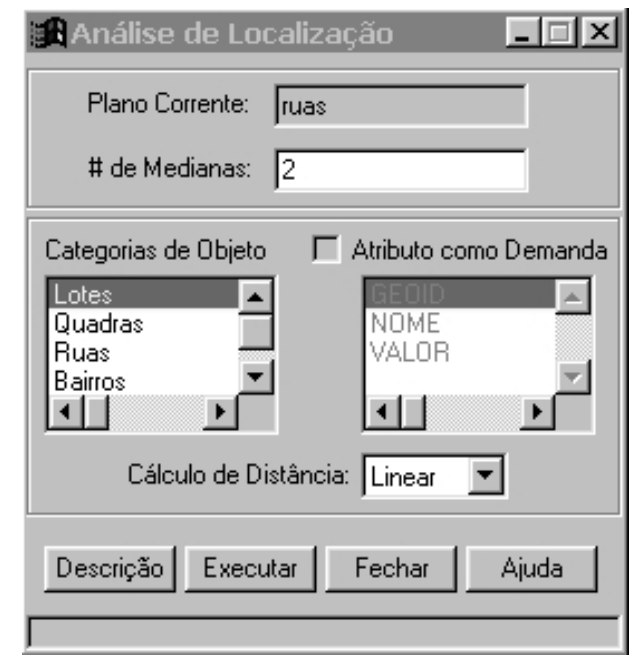

Figura 6 - Interface de diálogo para localização de medianas no SPRING.

O algoritmo para localização das medianas pode ser aplicado no SPRING em dados dos modelos temático, cadastral e de redes, da forma descrita a seguir:

- Para uso em um dado temático é necessário que a representação vetorial contenha pontos. As localizações espaciais dos pontos e a distância linear entre os mesmos são utilizados no processo de localização das medianas.

- Para o dado cadastral o procedimento de localização das medianas atua sobre uma determinada categoria de objetos selecionada. Todos os objetos desta categoria que estejam associados a uma representação pontual são utilizados na análise de localização, que usa a distância linear entre os pontos.

- Para o modelo de redes o modo de utilização é similar ao do modelo cadastral, com a diferença de que a distância entre os pontos pode ser escolhida entre linear, ou ser computada a partir da própria rede.

A Figura 6 mostra a interface para execução da função de localização de medianas no SPRING. O cálculo das medianas usa a área da informação que está visível no monitor. A partir de um plano de informação ativo, o usuário define o número de medianas a serem calculadas. Se o plano ativo corresponder a um dado temático esta é a única informação necessária a ser fornecida, sendo considerada a distância linear entre os pontos. Para o caso de dados cadastrais ou de redes, a lista de categorias de objeto fica ativa para que seja selecionado um tipo de objeto. Em princípio apenas objetos do mesmo tipo entram na análise de localização, podendo esta restrição não existir em versões futuras. O cálculo da distância entre os pontos corresponde à distância linear para os modelos temático e cadastral, enquanto que para o modelo de redes também está disponível selecionar que a distância seja calculada baseada na própria rede. Para o caso com restrições de capacidade, a interface apresenta a opção de se associar algum valor de demanda ou peso para os pontos em análise. Este valor pode ser obtido a partir de um atributo do objeto no banco de dados.

As Figuras 7 e 8 mostram os resultados da análise de localização em uma pequena região da cidade de São José dos Campos. Alguns objetos localizados em vértices da rede correspondem às possíveis localizações para instalação de algum tipo de atividade. Dado o número de medianas a se encontrar, o programa apresenta na tela os pontos correspondentes às medianas (representados por círculos) e associa os outros pontos à mediana mais próxima. Pode-se observar que os resultados considerando distâncias lineares e da rede não são necessariamente iguais. 


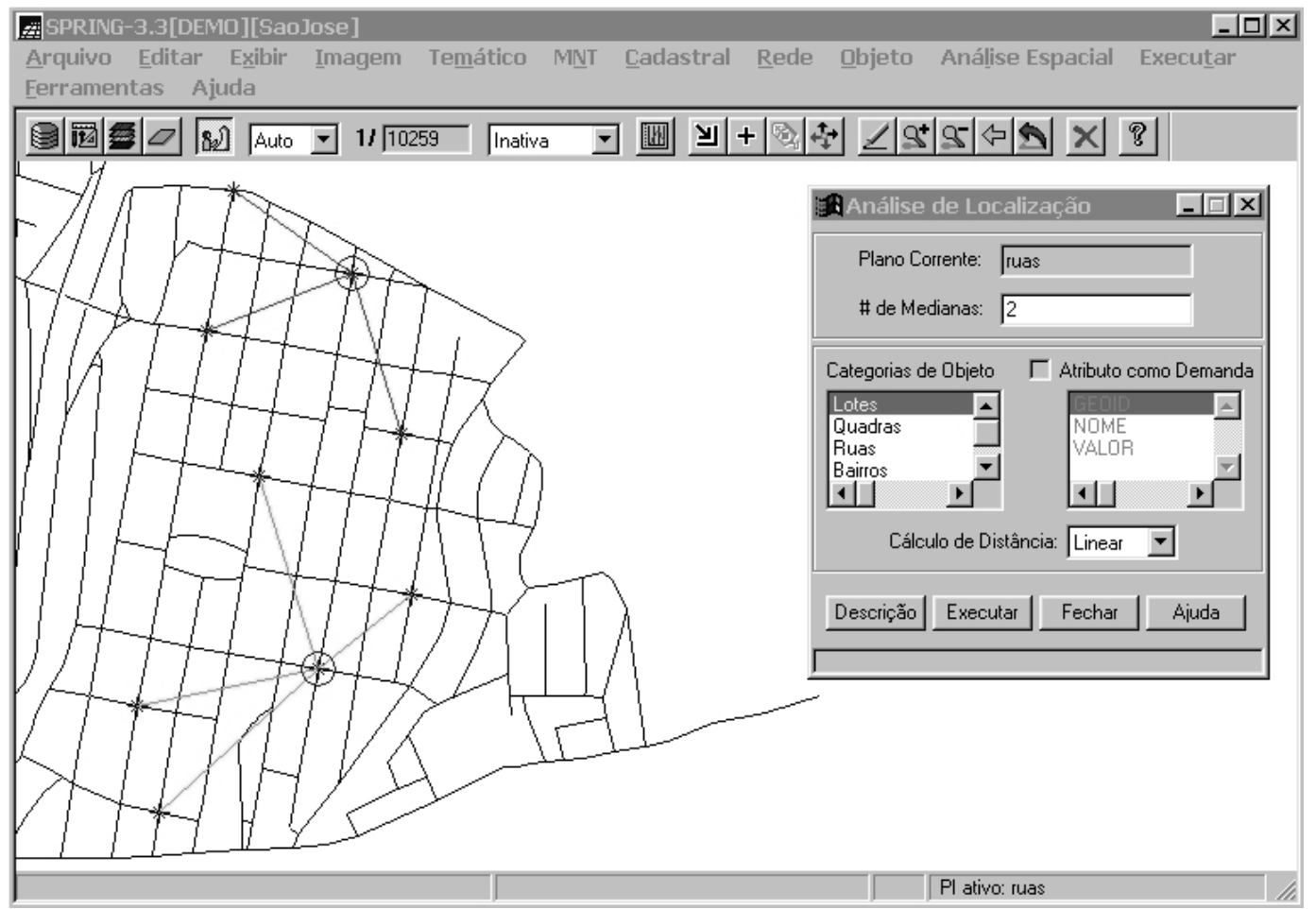

Figura 7 - Cálculo de medianas no SPRING usando distância linear.

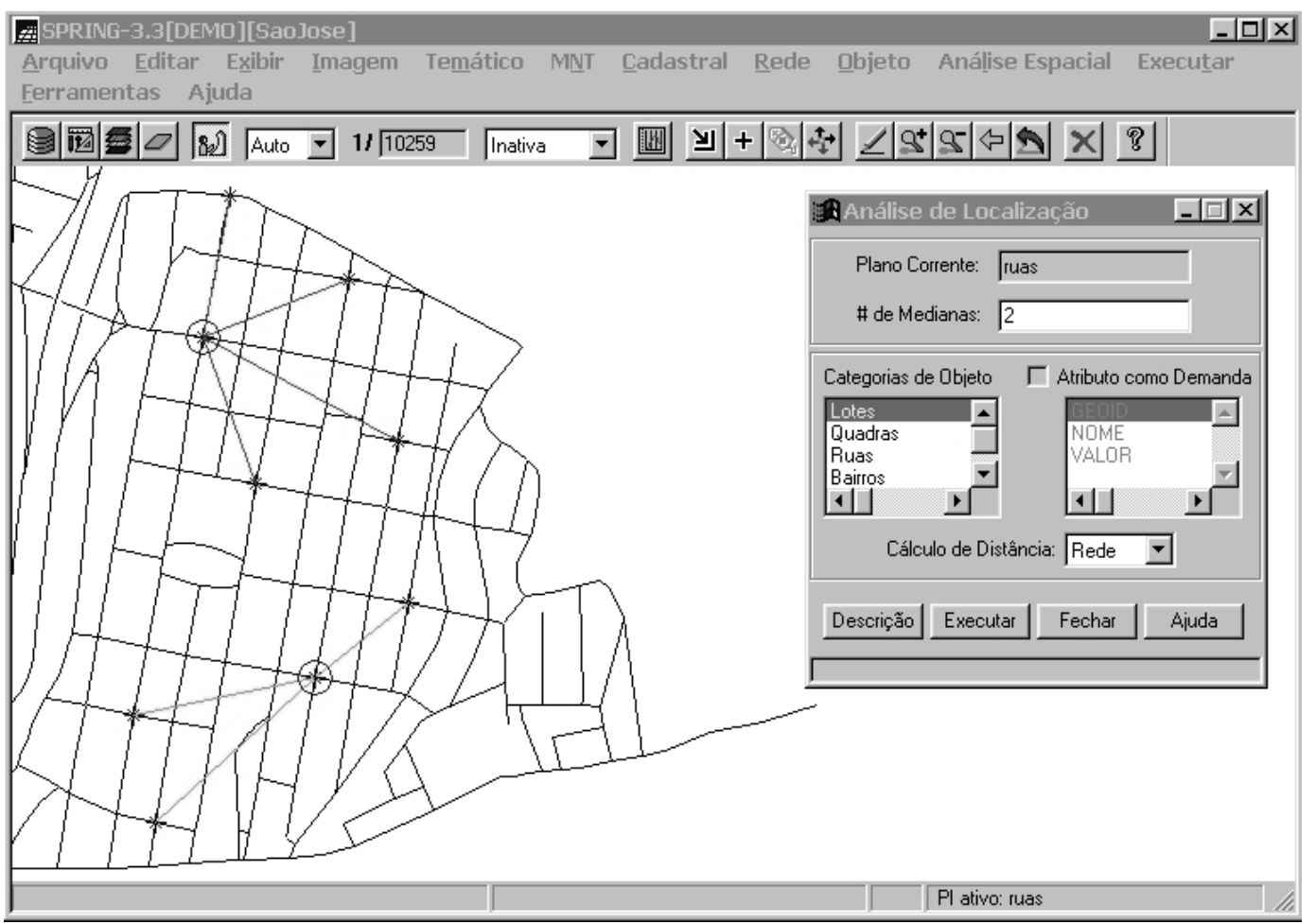

Figura 8 - Cálculo de medianas no SPRING usando distâncias da rede. 


\section{Testes Computacionais e Resultados}

$\mathrm{F}$ oram realizados alguns testes computacionais para testar a eficiência do algoritmo de $p$-medianas apresentado na seção 3. Neste trabalho foi utilizado um microcomputador Pentium MMX $233 \mathrm{MHz}$ com $128 \mathrm{MB}$ de RAM e os dados correspondem às quadras e ruas da região central da cidade de São José dos Campos.

Os resultados estão apresentados nas Tabelas 1 e 2. Nestas tabelas, todos os tempos computacionais excluem o tempo necessário para estabelecer a matriz de distâncias. As tabelas contêm:

- n: número de vértices da rede;

- p: número de medianas;

- LInf: valor da melhor solução dual obtida (limite inferior da solução ótima);

- LSup: valor da melhor solução viável obtida (limite superior da solução ótima);

- Gap: gap percentual de dualidade, ou seja, $100 \times($ LSup - LInf $) /$ LSup;

- Tempo: tempo computacional (em segundos).

Os resultados da Tabela 1 mostram que os valores para os gaps de dualidade são pequenos, demonstrando a efetividade do algoritmo de $p$-medianas para dados reais distribuídos geograficamente.

Os resultados com dados reais para o caso com restrições de capacidade compõem a Tabela 2. Como se pode observar, a complexidade do problema reflete-se nos tempos computacionais, sensivelmente mais elevados que os problemas sem restrições de capacidade de mesma dimensão. Os gaps de dualidade também se mantiveram baixos, confirmando a robustez da heurística também para este tipo de problema.
Os dados utilizados e os resultados obtidos estão disponíveis em http://www.lac.inpe.br/ ulorena/ArsigIndex.html.

\section{Conclusão}

$\mathrm{N}$ este trabalho discutiu-se a integração de modelos de $p$-medianas aos Sistemas de Informações Geográficas ArcView, da ESRI, e SPRING, em desenvolvimento no INPE. O código integrado a estes SIGs considera uma implementação recente da heurística lagrangiana/ surrogate, aplicada na resolução de problemas de localização e de atribuição.

A complexidade inerente ao problema de $p$-medianas com restrições de capacidade refletese nos tempos computacionais, mais elevados que o caso não capacitado com mesmo número de vértices. Apesar disso, os gaps de dualidade mantiveram-se baixos, comprovando a eficiência da heurística apresentada.

Os testes computacionais realizados usando dados do município de São José dos Campos demonstraram a efetividade do algoritmo proposto para utilização em Sistemas de Apoio à Decisão usando Sistemas de Informações Geográficas.

\section{Agradecimentos}

Os autores agradecem o apoio financeiro da FAPESP - Fundação para o Amparo à Pesquisa no Estado de São Paulo (proc. 96/04585-6). O primeiro, segundo e quarto autores agradecem também o apoio recebido do $\mathrm{CNPq}$ - Conselho Nacional de Desenvolvimento Científico e Tecnológico (proc. 350034/91-5, 302408/88-6 e 380646/99-4, respectivamente). 
Tabela 1 - Resultados dos Testes Computacionais: Caso sem Restrições de Capacidade.

\begin{tabular}{|c|c|r|r|r|r|}
\hline $\mathbf{n}$ & $\mathbf{p}$ & \multicolumn{1}{c|}{ LInf } & \multicolumn{1}{c|}{ LSup } & Gap & \multicolumn{1}{c|}{ Tempo } \\
\hline 324 & 5 & 122518,02 & 122518,02 & 0,000 & 4,72 \\
& 10 & 79250,84 & 79256,35 & 0,007 & 7,30 \\
& 20 & 54467,23 & 54533,11 & 0,121 & 7,33 \\
& 50 & 32094,13 & 32101,52 & 0,023 & 7,65 \\
& 108 & 18719,70 & 19683,61 & 4,897 & 7,84 \\
\hline 818 & 5 & 604883,69 & 605855,81 & 0,160 & 102,66 \\
& 10 & 382420,75 & 385371,44 & 0,766 & 97,48 \\
& 20 & 251540,45 & 251717,77 & 0,070 & 60,39 \\
& 50 & 146303,64 & 149251,13 & 1,975 & 43,73 \\
& 100 & 97763,44 & 98992,31 & 1,241 & 57,93 \\
& 150 & 75465,67 & 77440,57 & 2,550 & 66,19 \\
& 272 & 47481,36 & 50086,61 & 5,201 & 85,58 \\
\hline 3282 & 5 & 6381066,50 & 6381119,00 & 0,001 & 1699,88 \\
& 10 & 3911948,00 & 3914249,75 & 0,059 & 1548,43 \\
& 20 & 2342928,75 & 2350502,50 & 0,322 & 1520,00 \\
& 50 & 1288593,00 & 1308957,25 & 1,556 & 1106,45 \\
& 100 & 838007,63 & 841380,81 & 0,401 & 954,24 \\
& 500 & 322401,41 & 332954,84 & 3,170 & 1530,44 \\
& 1000 & 186532,23 & 194813,50 & 4,251 & 1606,07 \\
& 1141 & 164245,19 & 175905,27 & 6,629 & 1526,76 \\
\hline
\end{tabular}

Tabela 2 - Resultados dos Testes Computacionais: Caso com Restrições de Capacidade.

\begin{tabular}{|c|c|c|l|c|r|}
\hline $\mathbf{n}$ & $\mathbf{p}$ & LInf & LSup & Gap & Tempo \\
\hline 100 & 10 & 17246,53 & 17288,99 & 0,246 & 187,67 \\
200 & 15 & 33225,88 & 33426,04 & 0,599 & 4249,74 \\
300 & 25 & 45314,71 & 45364,30 & 0,109 & 4956,45 \\
300 & 30 & 40635,80 & 40635,91 & 0,000 & 3403,03 \\
402 & 30 & 61843,23 & 62000,23 & 0,253 & 41988,99 \\
402 & 40 & 52396,31 & 53225,30 & 1,558 & 9673,38 \\
\hline
\end{tabular}




\section{Referências Bibliográficas}

BEASLEY, J.E.: "Lagrangean Heuristics for Location Problems." European Journal of Operational Research, 65, p. 383-399, 1993.

BURROUGH, P.A.: Principles of Geographical Information Systems for Land Resources Assessment. Clarendon Press, Oxford, 1986.

CÂMARA, G.: Modelos, Linguagens e Arquiteturas para Bancos de Dados Geográficos. Tese de Doutorado (INPE), São José dos Campos, SP, 1995.

CHRISTOFIDES, N. \& BEASLEY, J.E.: "A tree search algorithm for the p-median problem." European Journal of Operational Research, 10, p. 196-204, 1982.

COOPER, L.: "Location-allocation problems." Operations Research, 11, p. 331-343, 1963.

DASKIN, M.: Network and Discrete Location: Models, Algorithms, and Applications. Wiley Interscience, New York, 1995.

DREZNER, Z. (ed.): Facility Location: A Survey of Applications and Methods. Springer-Verlag, New York, 1995.

DYER, M.E.: "Calculating surrogate constraints." Mathematical Programming, 19, p. 255-278, 1980.

ESRI: Avenue Customization and Application Development for ArcView. Environmental Systems Research Institute, Inc., Redlands, CA, 1996.

FISCHBECK, P.: "GIS: More than a Map." OR/MS Today, p. 42-45, Agosto 1994.

GALVÃO, R.D. \& RAGGI, L.A.: "A method for solving to optimality uncapacitated location problems." Annals of Operations Research, 18, p. 225-244, 1989.

GAREY, M.R. \& JOHNSON, D.S.: Computers and intractability: a guide to the theory of NP-completeness. W.H. Freeman and Co., San Francisco, CA, 1979.

GLOVER, F.: "Surrogate constraints." Operations Research, 16, p. 741-749, 1968.

HAKIMI, S.L.: "Optimum location of switching centers and the absolute centers and the medians of a graph." Operations Research, 12, p. 450-459, 1964.
HAKIMI, S.L.: "Optimum distribution of switching centers in a communication network and some related graph theoretic problems." Operations Research, 13, p. 462-475, 1965.

HANDLER, G. \& ZANG, I.: "A dual algorithm for the constrained shortest path problem." Networks, 10, p. 293-310, 1980.

HELD, M. \& KARP, R.M.: "The traveling-salesman problem and minimum spanning trees: part II." Mathematical Programming, 1, p. 6-25, 1971.

JARVINEN, P.J. \& RAJALA, J.: "A branch and bound algorithm for seeking the p-median." Operations Research, 20, p. 173-178, 1972.

KARWAN, M.L. \& RARDIN, R.L.: "Some relationships between Lagrangean and surrogate duality in integer programming." Mathematical Programming, 17, p. 320-334, 1979.

LORENA, L.A.N. \& LOPES, F.B.: "A surrogate heuristic for set covering problems." European Journal of Operational Research, 79, p. 138-150, 1994.

LORENA, L.A.N. \& NARCISO, M.G.: "Relaxation Heuristics for Generalized Assignment Problems." European Journal of Operational Research, 91, p. 600-610, 1996.

LORENA, L.A.N. \& SENNE, E.L.F.: “A Lagrangean/ Surrogate Heuristic for Uncapacitated Facility Location Problems." Em: Annals of the $8^{\text {th }}$ LatinIberian-American Congress on Operations Research and System Engineering, Anais do $28^{\underline{o}}$ Simpósio Brasileiro de Pesquisa Operacional, p. 854-859, Rio de Janeiro, Agosto 1996.

LORENA, L.A.N. \& SENNE, E.L.F.: "Improving traditional subgradient scheme for Lagrangean relaxation: an application to location problems." International Journal of Mathematical Algorithms, 1, p. 133-151, 1999.

MARTELLO, S. \& TOTH, P.: Knapsack problem: Algorithms and computer implementations. John Wiley \& Sons, New York, 1990.

MINOUX, M.: "Plus courts chemins avec constraints: Algorithmes et applications." Annals of Telecommunications, 30, p. 383-394, 1975. 
NARCISO, M.G. \& LORENA, L.A.N.: "Lagrangean/ surrogate relaxation for generalized assignment problems." European Journal of Operational Research, 114, p. 165-177, 1999.

NEEBE, A.W.: "A branch and bound algorithm for the p-median transportation problem." Journal of the Operational Research Society, 29, p. 989-995, 1978.

PARKER, R.G. \& RARDIN, R.L.: Discrete Optimization. Academic Press, New York, 1988.

SENNE, E.L.F. \& LORENA, L.A.N.: "Lagrangean/ Surrogate Heuristics for Facility Location Problems." Em: Annals of EURO XV - INFORMS XXXIV Joint International Meeting. Barcelona, Espanha, p. 128, Julho 1997.
SENNE, E.L.F. \& LORENA, L.A.N.: "Lagrangean/ surrogate heuristics for p-median problems." Em: M. LAGUNA and J.L. GONZALEZ-VELARDE (eds.): Computing Tools for Modeling, Optimization and Simulation: Interfaces in Computer Science and Operations Research, Kluwer Academic Publishers, p. 115-130, 2000.

SPRING: Sistema de Processamento de Informações Georeferenciadas. INPE, São José dos Campos, SP, <http://www.dpi.inpe.br/spring>, 1998.

TAILLARD, E.D.: "Heuristic methods for large centroid clustering problems." Technical Report IDSIA96-96, IDSIA, 1996.

TEITZ, M.B. \& BART, P.: "Heuristic Methods for Estimating the Generalized Vertex Median of a Weighted Graph." Operations Research, 16, p. 955-961, 1968.

\title{
INTEGRATION OF LOCATION MODELS TO GEOGRAPHICAL INFORMATION SYSTEMS
}

\begin{abstract}
The $\mathrm{p}$-median problems deal with decisions of locating $p$ facilities (medians) in a network, minimizing the sum of all distances from each vertex to its nearest facility. If demand information is available for each vertex of the network, then values on the capacity of each facility may be present (capacitated p-median problems). Facility location models have been proposed as decision making tools, mainly when geographic information systems (GIS) can be used to capture, store and analyze the data of the problems. In this work we present the integration of a p-median algorithm to both ArcView, a GIS by ESRI (Environmental Systems Research Institute, Inc.), and SPRING, a GIS developed by INPE (National Institute for Space Research). The computer programme that has been integrated to these geographic information systems implements a recent approach of Lagrangean/surrogate heuristic which uses location-allocation heuristics in order to search for the prime feasibility of intermediate dual solutions. The paper presents some computational tests which have been conducted with real data from the city of São José dos Campos, representing problems with up to 3280 vertices and 1141 medians, for the uncapacitated problem.
\end{abstract}

Key words: Facility Location, Geographical Information Systems, Lagrangean Heuristics. 